\title{
Application of negative group delay active circuits to the design of broadband and constant phase shifters
}

\author{
B. Ravelo, M. Le Roy and A. Pérennec
}

ABSTRACT: The new phase-shifter configuration described in this report uses a negative group delay (NGD) active circuit. In this topology, a classical transmission line is set in cascade with an NGD circuit whose phase slopes are alike, but opposite, to get a constant and broadband phase shift. The proposed approach was validated through the design and measurement of a phase shifter, which exhibited a constant phase of $90^{\circ} \pm 5^{\circ}$ over a $75 \%$ relative bandwidth around $1.6 \mathrm{GHz}$. Moreover, as the group delay of the NGD circuit compensated the transmission line one, the overall circuit group delay was kept to a small value in the operating frequency band.

Key words: phase shifters; negative group delay; active circuits

Authors' affiliations:

B. Ravelo, M. Le Roy and A. Pérennec

LAB-STICC, Université de Bretagne Occidentale, FRE CNRS 3167, CS93837,

F- 29238 Brest cedex 3, France

E-mail : Marc.LeRoy@univ-brest.fr

Fax : (33) 298016395 


\section{INTRODUCTION}

In [1], Lucyszyn and co-workers were the first to propose a negative group delay synthesiser operating at microwave frequencies. This narrowband and tunable microwave circuit operates in reflection, needs a coupler to pass in transmission and exhibits high negative group delay (NGD) values together with very high losses. More recently, many configurations with negative group delays have been described in the literature. Indeed, negative group velocity and, thus, NGD are known to be experienced in some metamaterials and, in particular, in left-handed (LH) lines with resonant cells [2]. Such lines are built with a high-pass L-C network cascaded with a series RLC shunt cell. In this case, NGD values are always associated with high losses. In physics, abnormal group velocities have also been evidenced in several experiments [3]. To facilitate the study of this intriguing phenomenon, many low frequency circuits generating simultaneously NGD and gain have been implemented with operational amplifiers and lumped components [4]. But, their use at higher frequencies is limited because of the components bandwidths. So, in [5]-[6], the authors showed by theory and by experiments that the active topology presented in Figure $1(a)$ is able to simultaneously provide gain and NGD at microwave frequencies and for different bandwidths. Moreover, as the field effect transistor (FET) is simply modelled (Figure $1(b))$ by the transconductance, $g_{m}$, and the drainsource resistor, $R_{d s}$, synthesis equations were easily extracted [5]-[6]. When dealing with localised circuits, the notion of group delay has to be used instead of group velocity. The group delay is defined as the derivate of the $S_{21}$ parameter phase response versus the angular frequency, $\omega$ :

$$
\tau(\omega)=-\frac{\partial \phi_{S_{21}}}{\partial \omega}
$$


An NGD is, therefore, induced by a positive phase slope, which can be obtained thanks to this topology. By way of consequence it sounded to us worth implementing such an active NGD circuit in order to design phase shifters with a constant transmission phase.

\section{FLAT PHASE PRINCIPLE}

Our approach should be strongly distinguished from recent applications with LHmedia [7]-[8]. Indeed, in these applications, a transmission line (low-pass topology) with a negative phase slope is associated with a reverse high-pass topology (LH-line) with an identical negative phase slope but with a positive starting phase to get a constant phase difference at the design frequency. By associating branches with such parallel phase slopes [7] or tuning between high- or low-pass topologies [7] and for size reductions or bandwidth-broadening considerations [7]-[8], LH-lines have found applications in power divider/splitters, baluns, couplers or phase shifters. Compared to these proposals, we designed a circuit with a constant and frequencyindependent phase instead of a constant phase shift difference. Let $\tau$ be the constant group delay; from Eq. 1 the linear transmission phase, $\phi(f)$ is given by:

$$
\phi(f)=-2 \pi \tau f+\phi(0)
$$

Figure 2 illustrates the principle presented here: in short, it consists in the cascading of a classical line (referred as PGD for positive group delay), whose phase slope is negative, by an NGD circuit with a positive and opposite phase slope. In the frequency band within $f_{1}$ and $f_{2}$, the PGD and NGD device phases are respectively expressed as:

$$
\begin{aligned}
& \phi_{P}(f)=-2 \pi \tau_{P}\left(f-f_{1}\right)+\phi_{P}\left(f_{1}\right) \\
& \phi_{N}(f)=-2 \pi \tau_{N}\left(f-f_{1}\right)+\phi_{N}\left(f_{1}\right)
\end{aligned}
$$


For well-matched devices, once cascaded, the phase of the whole is:

$$
\phi_{T}(f)=\phi_{P}(f)+\phi_{N}(f)=-2 \pi\left(\tau_{P}+\tau_{N}\right)\left(f-f_{1}\right)+\left[\phi_{P}\left(f_{1}\right)+\phi_{N}\left(f_{1}\right)\right]
$$

The overall transmission phase, $\phi_{T}$, (in thick curve in Figure 2(b)) is then constant in the specified frequency band for opposite phase slopes or opposite group delay values:

$$
\begin{aligned}
& \tau_{N}=-\tau_{P} \\
& \phi_{T}(f)=\phi_{P}\left(f_{1}\right)+\phi_{N}\left(f_{1}\right)=C^{t e}
\end{aligned}
$$

This total phase value is the sum, at the frequency, $f_{1}$, of those of the two circuit parts, and it can simply be adjusted by varying the line length. As depicted in Figure 2(b), the resulting theoretical group delay obtained by application of Eq.6 is low in the operational bandwidth whatever the target phases.

\section{DESIGN OF FREQUENCY-INDEPENDENT PHASE SHIFTER AND RESULTS}

A prerequisite to the design process was the calculation of the RLC component values of the NGD circuit of Figure 1 from the synthesis equations [5] extracted from Eqs. (8) and (9):

$$
\begin{aligned}
& S_{21}\left(\omega_{0}\right)=\frac{-2 R Z_{0} g_{m} R_{d s}}{R_{d s} R+Z_{0}\left(R+R_{d s}\right)} \\
& \tau\left(\omega_{0}\right)=\frac{-2 L Z_{0} R_{d s}}{R \cdot\left[R R_{d s}+Z_{0}\left(R+R_{d s}\right)\right]}
\end{aligned}
$$

Relation (9) guarantees that the group delay is always negative at the RLC series resonance frequency, $\omega_{0}$. A direct cascading of circuits with opposite phase slopes in order to get a constant phase value is valid only when the two devices are well matched. So, here, the NGD circuit was matched at the input by the shunt resistor, 
$R_{m 1}$, and at the output by the series resistor, $R_{m 2}$; then, it was cascaded with two 50$\Omega$ transmission lines, TL1 and TL2, set at the input and output to get the frequencyconstant phase. At first, the circuit used for simulations was the one described in figure 3(a)) with the given values for components and substrate and with the Sparameters available for this FET. Then, electromagnetic simulations of the layout shown in Figure $3(\mathrm{~b})$ were run with ADS Momentum software prior to the final optimisation of the line lengths. Figure 4 compares the frequency responses obtained by EM simulations and experiments. It shows (Figure 4.a), a $90^{\circ} \pm 5^{\circ}$ transmission phase shift from 1.08 to $2.42 \mathrm{GHz}$. One should note that the group delay is even slightly negative from 1.4 to $2 \mathrm{GHz}$. On the other hand, between 1.08 and $2.42 \mathrm{GHz}$, the measured $S_{21}$ magnitude lies within 2 and $-2 \mathrm{~dB}$. Conversely, input and output return losses are better than in simulations. It is worth pointing out that every constant phase values within $+180^{\circ}$ and $-180^{\circ}$ can be obtained, and among them, the negative values require smaller line lengths on condition the number of active cells is even (initial NGD-circuit phase value at $0^{\circ}$ instead of $180^{\circ}$ ).

\section{CONCLUSION}

We designed and fabricated an active phase shifter with a flat and constant phase over a broad frequency band; experimental results confirmed the expected behaviour. To get this frequency-independent phase, an active NGD circuit with a positive phase slope was cascaded with a classical transmission line. As the proposed phase shifter topology provides a constant transmission phase and not a phase shift, it can be readily distinguished from existing approaches. Moreover, this flat phase induced a low and rather constant group delay value whatever the target phase. Wider frequency bands can be achieved by cascading stages with different 
NGD operating frequency bands as proposed in [6] and such circuits can operate simultaneously as phase shifter and pulse compressor in UWB devices for example. This topology also offers opportunities to build tunable phase shifters by replacing either the capacitor with a varactor or the inductance with an active inductor [8] or even both of them. Moreover, to overcome the frequency limitation problem of localised components, implementation of LC localised components in distributed circuits is scheduled. 


\section{References}

1 S. Lucyszyn, I.D. Robertson, and A.H. Aghvami, Negative group delay synthesiser, Electronic Letters 29 (1993), 798-800.

2 O.F. Siddiqui, S.J. Erickson, G.V. Eleftheriades, and M. Mojahedi, Timedomain measurement of negative group delay in negative-refractive-index transmission-line metamaterials, IEEE Trans. Microw. Theory Tech. 52 (2004), 1449-1454.

3 R. A. Shelby, D. R. Smith, and S. Schultz, Experimental verification of a negative index of refraction, Science, 292, $\mathrm{n}^{\circ} 5514,(2001), 77-79$.

4 T. Nakanishi, K. Sugiyama, and M. Kitano, Demonstration of negative group delays in a simple electronic circuit, American Journal of Physics 70, issue 11 (2002), 1117-1121.

5 B. Ravelo, A. Pérennec, M. Le Roy, and Y.G. Boucher, Active microwave circuit with negative group delay, IEEE Microw. Wirel. Compon. Lett., 17, issue 12 (2007), 861-863.

6 B. Ravelo, A. Pérennec, and M. Le Roy, Broadband balun using active negative group delay circuit, Europ.. Mic. Conf. (2007), 466 - 469.

7 Lee Cheng-Jung, Kevin M. K. H. Leong, and T. Itoh, Broadband quadrature hybrid design using metamaterial transmission line and its application in the broadband continuous phase shifter", IEEE MTT-S Int. Microw. Symp. (2007), $1745-1748$.

8 M. A. Y. Abdalla, K. Phang, G.V. Eleftheriades, A 0.13- $\mu \mathrm{m}$ CMOS phase shifter using tunable positive/negative refractive index transmission lines", IEEE Microw. Wirel. Compon. Lett., 16, issue 12, (2006) 705 - 707. 


\section{Figure captions}

Figure $1 \quad$ NGD active cell and its equivalent model.

Figure 2 Principle of the NGD phase-shifter: block diagram (a) and the corresponding ideal phases and group delays (b) ( $Z_{0}$ : reference impedance port).

Figure 3 Schematic (a) of the negative group delay phase shifter with $R_{m 1}=51 \Omega, R_{m 2}=22 \Omega, R=33 \Omega, L=4.3 \mathrm{nH}, C=1 \mathrm{pF}, R_{b}=1 \mathrm{k} \Omega, L_{b}=1 \mu \mathrm{H}$ and $C_{b}$ $=22 \mu \mathrm{F}, T L 1, T L 2\left(Z_{c}=50 \Omega, d_{T L 1+T L 2}=10.2 \mathrm{~mm}\right), F E T: E C-2612\left(g_{m}=98.14 \mathrm{mS}\right.$ and $R_{d s}=116.8 \Omega$ ) and the corresponding layout (b) on an FR-4 substrate: $\varepsilon_{r}=4.3$, $h=508 \mu \mathrm{m}$, size: $20.7 \mathrm{~mm} \times 20.5 \mathrm{~mm}$. Bias networks are in thin lines $\left(\mathrm{V}_{\mathrm{ds}}=3 \mathrm{~V}, \mathrm{I}_{\mathrm{ds}}=\right.$ $30 \mathrm{~mA})$.

Figure 4 Simulations and measurements of the phase shifter: $S_{21}$ phase and group delay (a) and $S_{21}$ magnitude and return losses (b) 
Figure 1
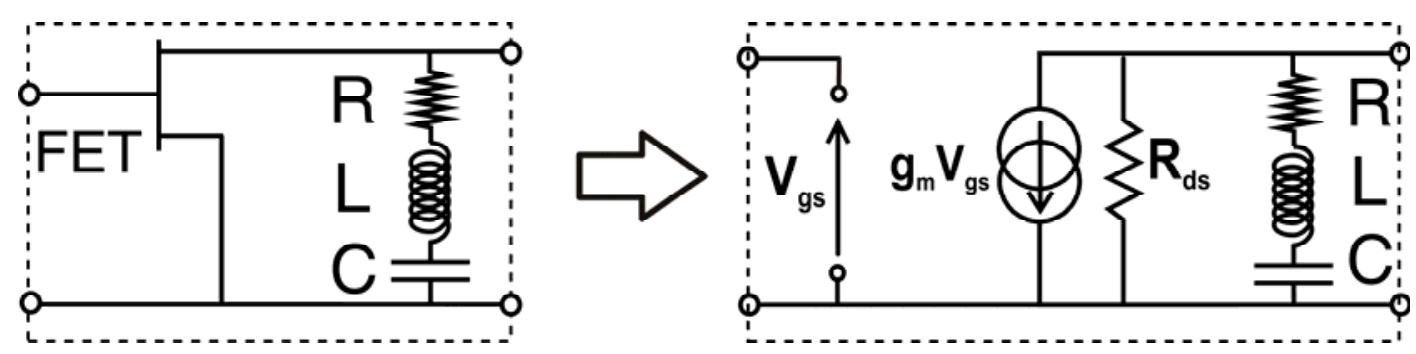
Figure 2

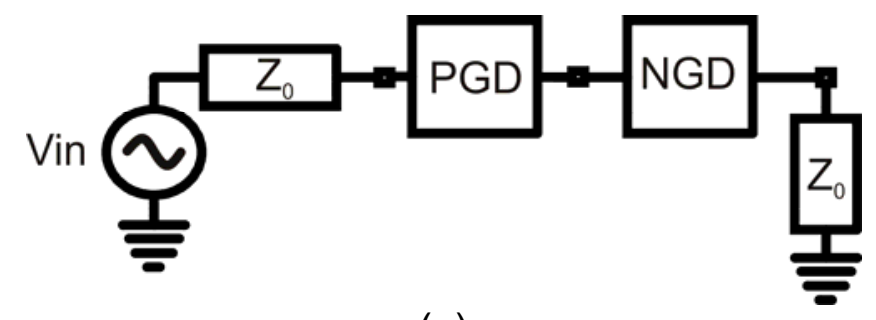

(a)

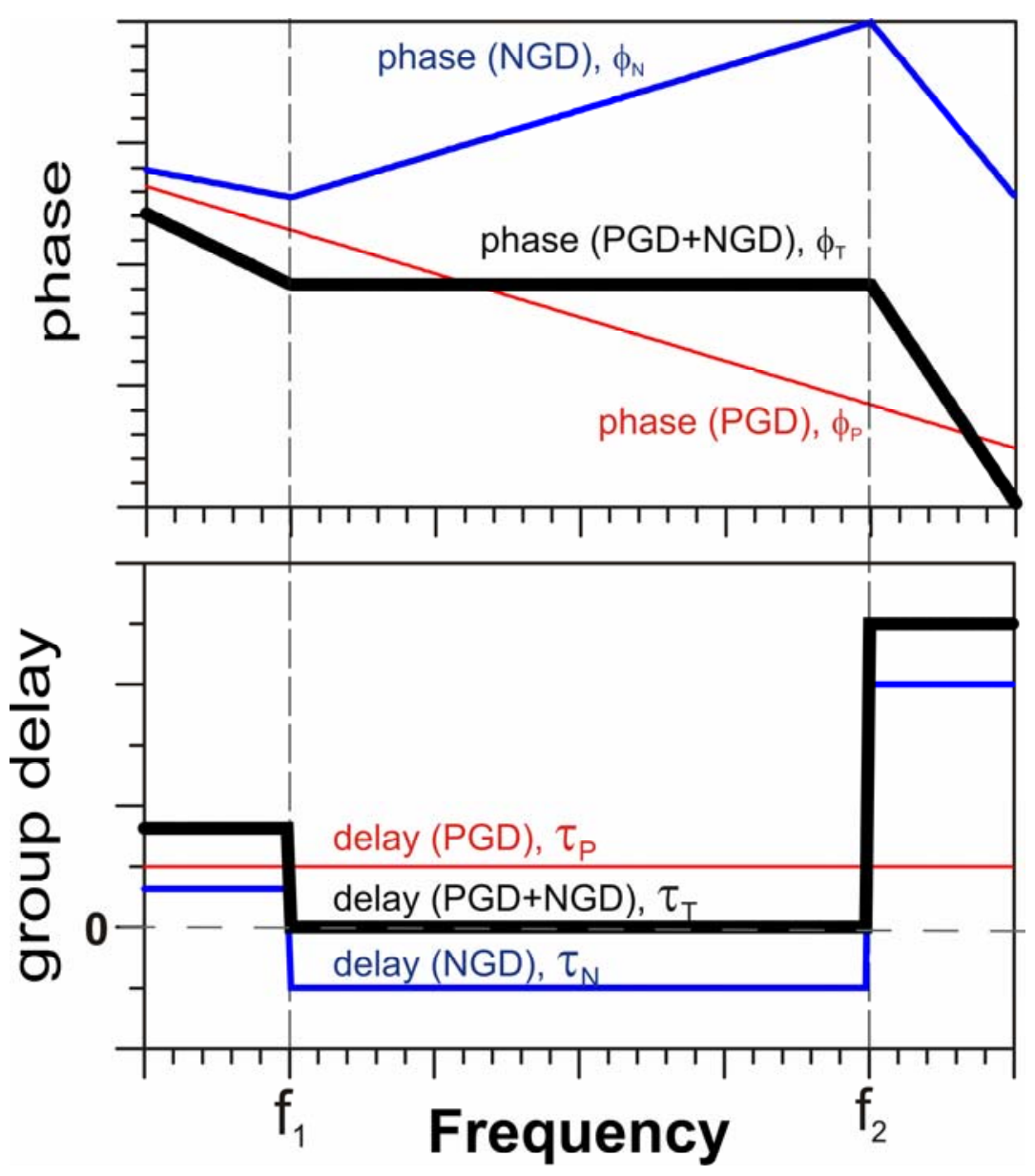

(b) 
Figure 3

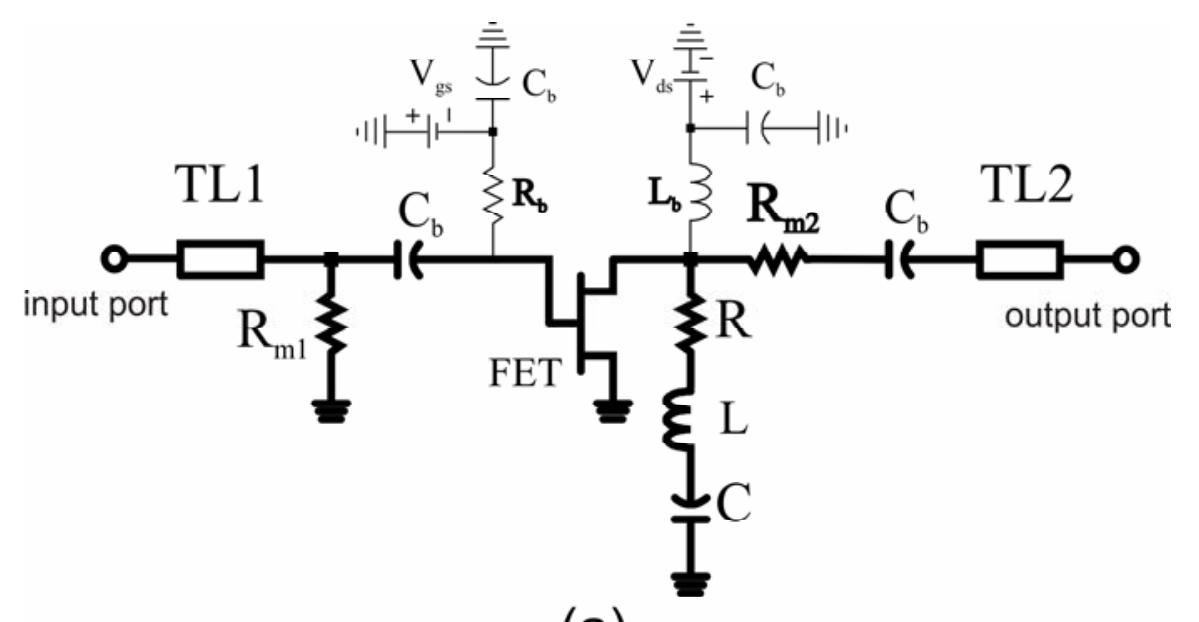

(a)

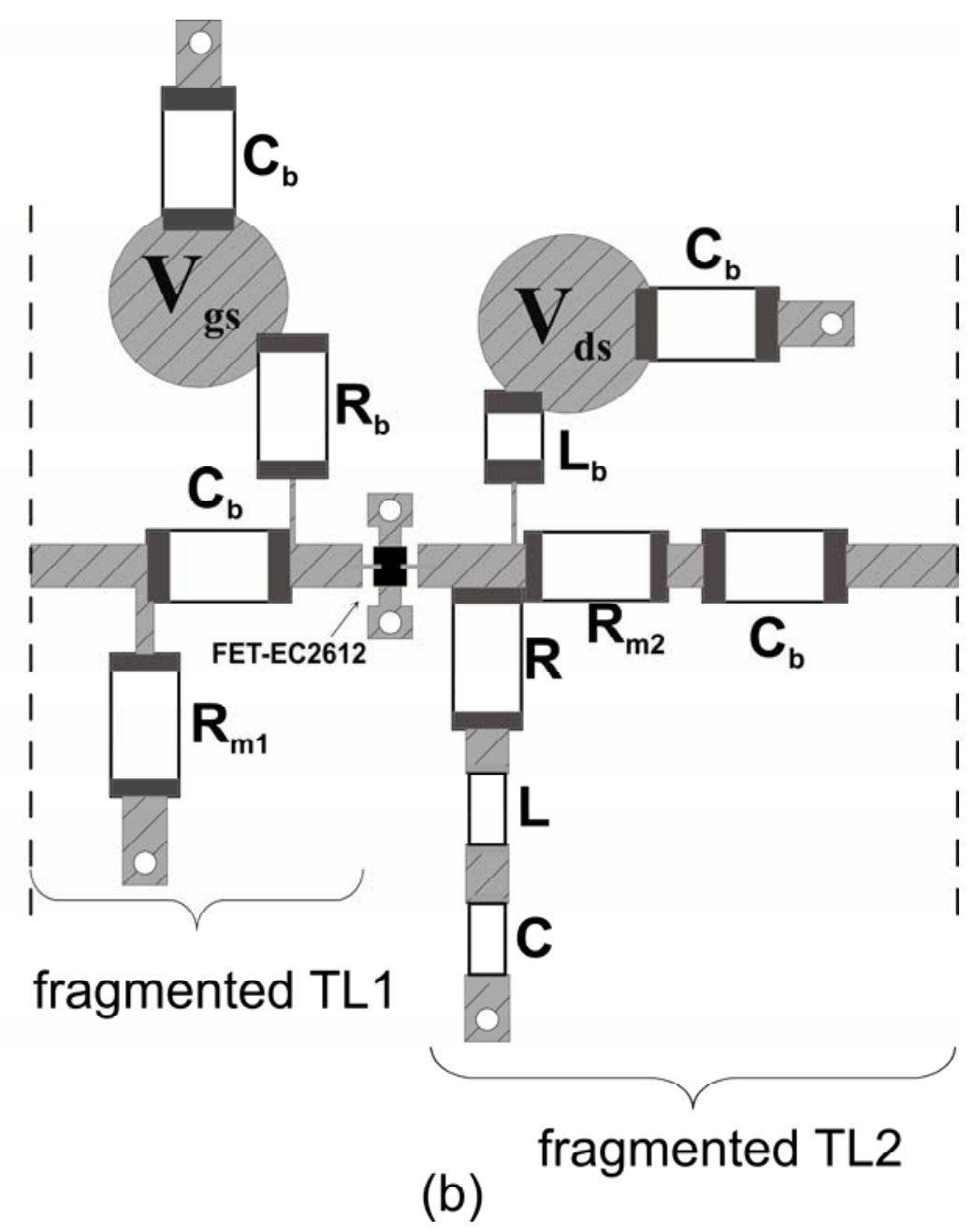

Figure 4-a 


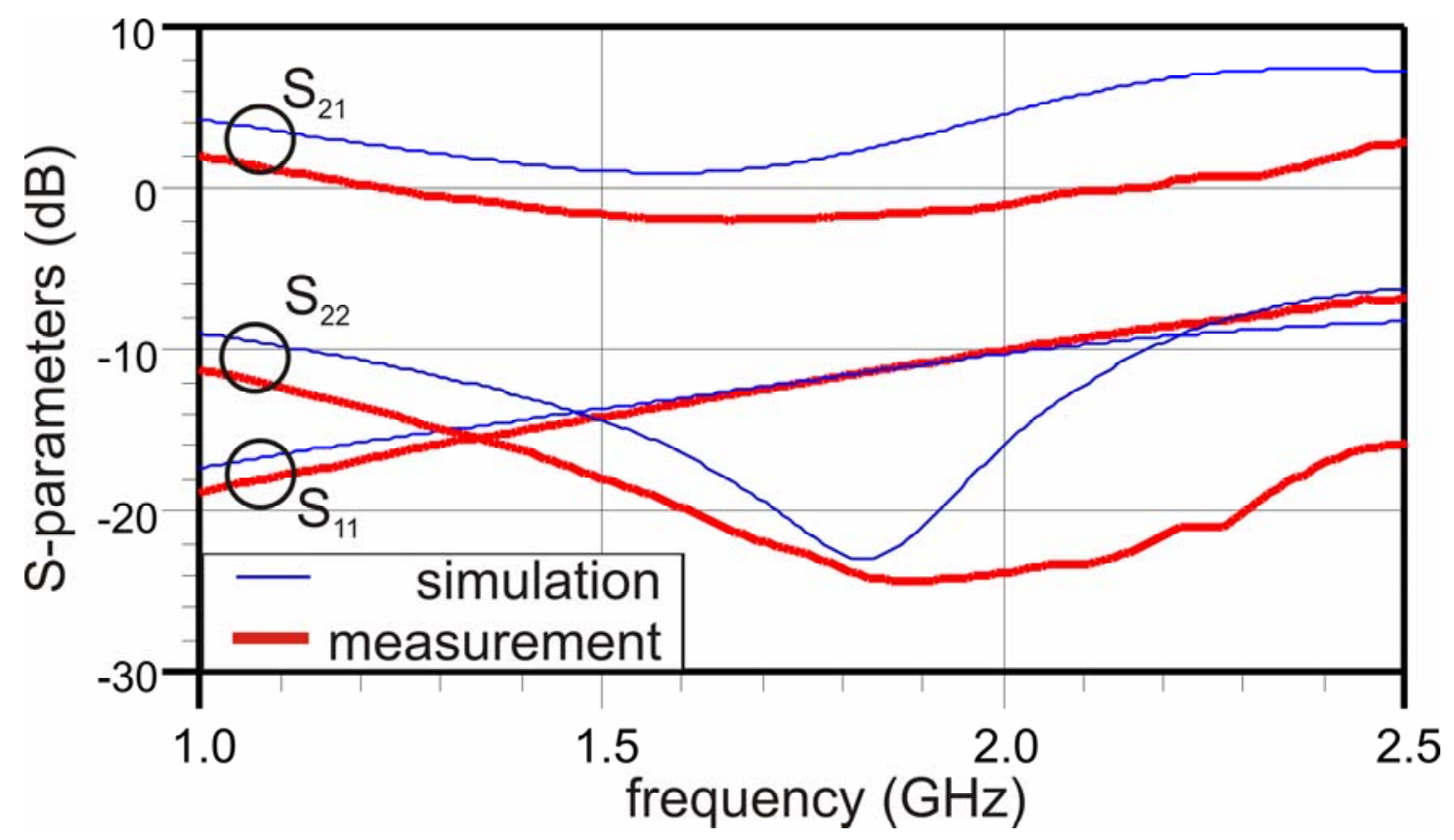

Figure 4-b

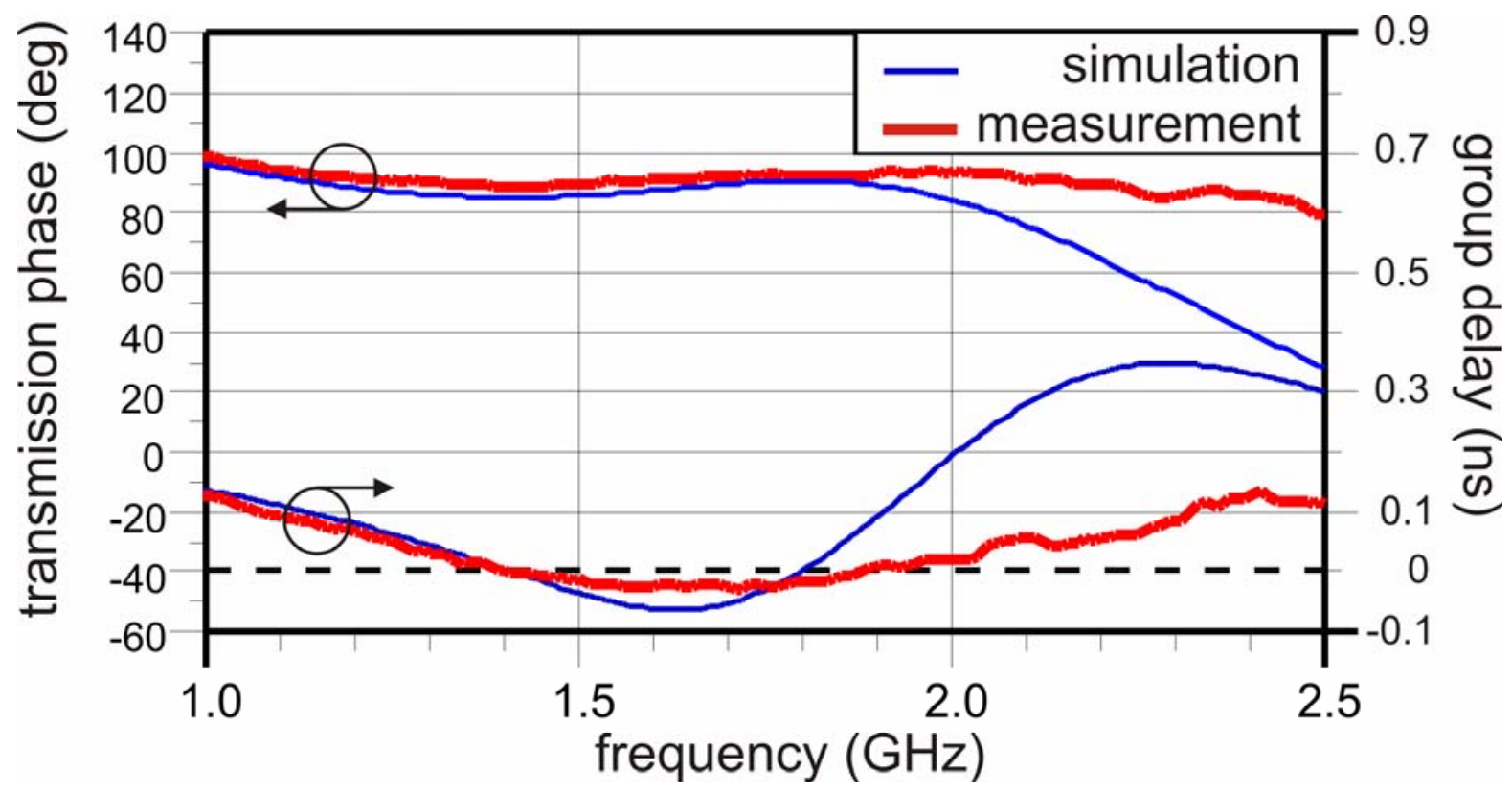

